\title{
Analysis and Adaptation of Integration Time in PMD Camera for Visual Servoing
}

\author{
Pablo Gil, Jorge Pomares, Fernando Torres \\ Physics, Systems Engineering and Signal Theory Dept. \\ University of Alicante \\ Alicante, Spain \\ e-mail: Pablo.Gil@ua.es
}

\begin{abstract}
The depth perception in the objects of a scene can be useful for tracking or applying visual servoing in mobile systems. 3D time-of-flight (ToF) cameras provide range images which give measurements in real time to improve these types of tasks. However, the distance computed from these range images is very changing with the integration time parameter. This paper presents an analysis for the online adaptation of integration time of ToF cameras. This online adaptation is necessary in order to capture the images in the best condition irrespective of the changes of distance (between camera and objects) caused by its movement when the camera is mounted on a robotic arm.
\end{abstract}

Time-of-flight cameras; integration time; image sensors; visual servoing; depth measurement; distance measurement

\section{INTRODUCTION}

The perception of an environment is a fundamental task, above all, for the object detection and characteristics extraction processes needed to guide a robot. These processes are required for its pose estimation and collision avoidance in real time. The time-of-flight (ToF) cameras are an alternative to standard techniques (such as laser scanners, stereoscopic vision, photogrammetry) where 3D information must be obtained. In the last years, several studies about calibration of ToF cameras and estimation of the distance errors have been published [1-4]. These researches are useful whenever the aim is to compute the distance in static scenes or make image processing. In this case, the distance between camera and target does not change. Therefore, it is not necessary to modify camera parameters, such as integration time, to adapt the range image captured. However, in the context of mobile robotics, where the scene is dynamic and the distance among the objects and the camera are continuously changed, the integration time of the camera must be adapted in order to improve the quality of the range images captured and to diminish the error. Two recent studies are remarkable in this context: In [5], a CSEMSwissrange camera is analyzed and in [6], a PMD camera is analyzed. In both cases, they are used for the navigation of a mobile robot in an environment with different objects. On the one hand, an integration time adaptation method based on the intensity parameter or luminance computed by the camera is proposed in [5]. On the other hand, the authors of [6] propose an algorithm based on amplitude parameter against [5], where the algorithm is based on intensity to be used in highly dynamic scenes. In this case, the time integration adaptation is not good because it is depending on illumination conditions and reflectance.

In this paper, a new approach based on amplitude parameter is used for integration time adaptation. In contrast with [6], the range of working distance analyzed is between 0.25 and 1 meter for the application of visual servoing.

This paper is organized as follows: In Section 2, the operation principle of ToF cameras and the PMD camera features are described. In Section 3, the PMD camera's behavior for a working distance smaller than $1 \mathrm{~m}$ is analyzed. In addition, an analysis for computing the threshold which defines critical working distance is developed. Finally, in Section 4, two ways (off-line and on-line process) are presented to compute the integration time considering static objects located at different distances from the camera in dynamic scenes where there is movement.

\section{MODEL AND PARAMETERS OF 3D-TOF}

Nowadays, the two main companies of 3D-ToF range cameras are PMDTechnologies and CSEM. These manufacturers present several cameras which acquire distance data using the time-of-flight principle, with invisible modulated near-infra-red (NIR) light, such as PMD19k or CamCube, Swissrange SR2 or SR3000. A specifications and behaviour comparison of these cameras is avaible in [5][7]. In this paper, the used camera is a PMD19k which provides grayscale images of 160x120 for classical image processing algorithms. In addtion, it provides distance (depth) and amplitude values for each pixel of those images. The wavelength of its NIR light is $870 \mathrm{~nm}$ and it can capture up to $15 \mathrm{fps}$ with a depth resolution of $6 \mathrm{~mm}$. In the experiments presented here, the camera is connected by Ethernet and it is programmable by SDK for Windows. Furthermore, it can be connected by Firewire interface and programmed for Linux. The ToF camera technology is based on the principle of modulation interferometry [7]. The scene is illuminated with NIR light and this light is reflected by the objects in the scene. The difference between both signals, emitted and reflected, causes a phase delay that is detected for each pixel and used to estimate the distance value. Thus, the ToF camera provides $2 \mathrm{D}$ depth information of dynamic or static scenes irrespective of the object's features. Given the speed 
light, $\mathrm{c}$, the frequency modulation, $\omega$, the correlation between signals for four internal phase delays, $\mathrm{r}_{0}\left(0^{\circ}\right), \mathrm{r}_{1}\left(90^{\circ}\right)$, $r_{2}\left(180^{\circ}\right), r_{3}\left(270^{\circ}\right)$, the camera computes the phase delay, $\varphi$, the amplitude, a, and the distance, $\mathrm{d}$, as follows:

$$
\begin{gathered}
\varphi=\arctan \left(\frac{r_{1}-r_{3}}{r_{0}-r_{2}}\right) \\
a=\frac{\sqrt{\left(r_{1}-r_{3}\right)^{2}+\left(r_{0}-r_{2}\right)^{2}}}{2} \\
d=\frac{c \varphi}{4 \pi \pi}
\end{gathered}
$$

\section{ANALYSIS OF RANGE IMAGES}

The PMD camera has two adjustable parameters: the modulation frequency and the integration time. In order not to change the original calibration determined by the manufacturer, only the integration time has been adjusted and the modulation frequency considered is the constant recommended by the manufacturer, $\omega=20 \mathrm{Mhz}$.

The integration time, $t$, is the most important intrinsic parameter of ToF cameras. The stability of the obtained measurements, such as intensity and distance of the range images, depends on the integration time. Generally, this parameter is previously adjusted in relation to each scene depending on the distance of the objects which appear in the environment (offline process). When a ToF camera is mounted at the end-effector of a robotic arm for visual servoing applications, this parameter has to be on-line adjusted to minimize the error in the computed depth. If this parameter is suitably determined, the image features are extracted in the best condition and so the quality of captured grayscale image is guaranteed.

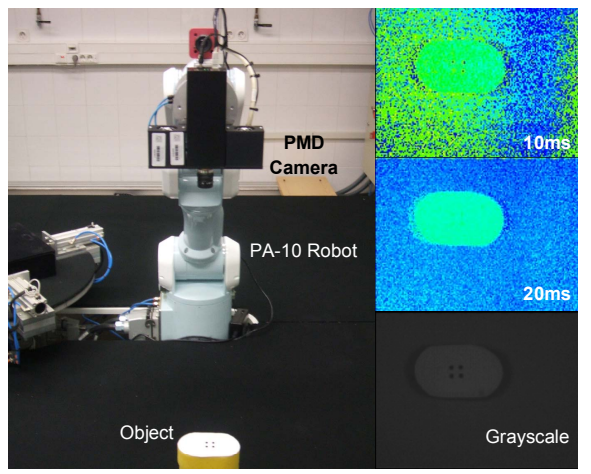

Figure 1. Eye-in-hand system and captured images using difference integration times.
Some experiments have been done in order to observe the evolution of the distance measured by the camera when the integration time changes. They involve acquiring some images of the same object over the same background positioned to different distances from the camera mounted at the robot effector (Fig. 1). The backboard is a black table which ensures a low reflectivity at the borders of the object.

In addition, the ambient light is controlled with power regulator in order to limit the interference with the NIR of the camera. In these experiments, the mean intensity, $\mathrm{i}_{\mathrm{m}}$, and the mean distance, $\mathrm{d}_{\mathrm{m}}$, are computed for each image from the information of each pixel provided by camera (Fig. 2). As Fig. 2 shows, if the time integration is small, the distance and intensity computed is unstable and untrustworthy. In the same way, if the time integration is great an oversaturation phenomenon is sometimes caused in the signal which determines the distance and intensity. Normally, this phenomenon only appears when the distance measured between scene and camera is below a threshold (close objects $<0.4 \mathrm{~m}$, it is verified in Section 4 ).
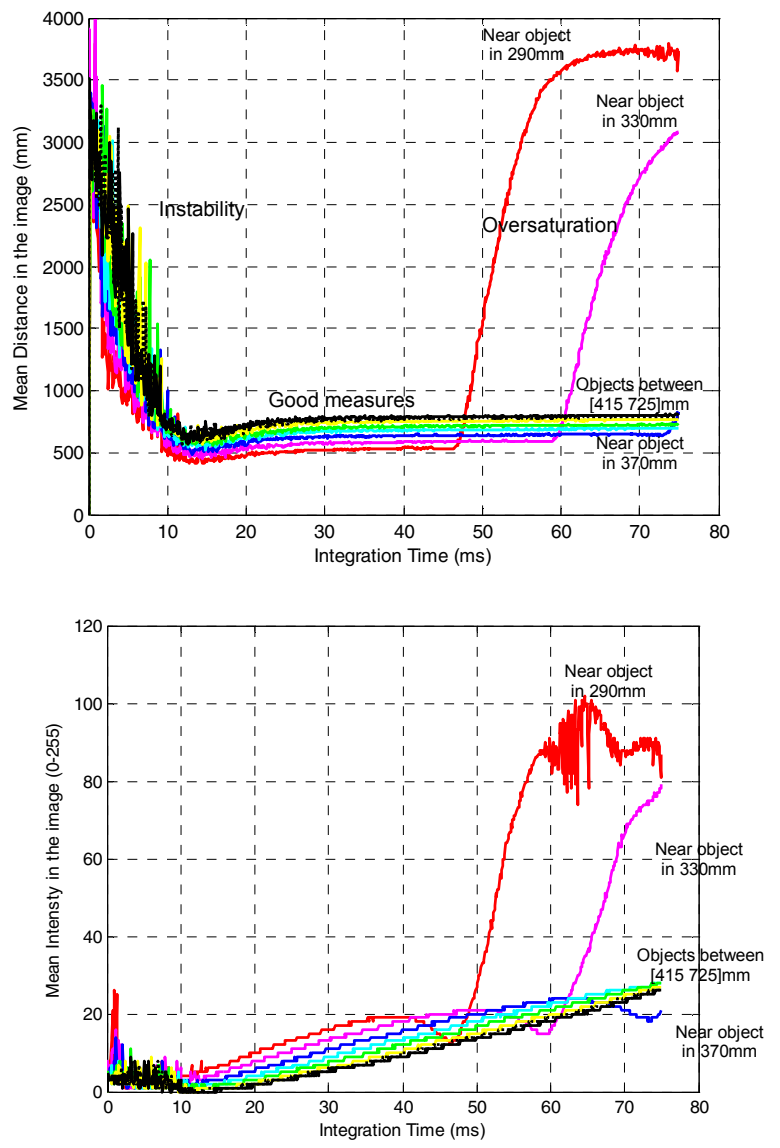

Figure 2. Evolution of mean distance, $d_{m}$, and intensity of the range images using some distances between camera and target.

However, the distances are correctly computed when the objects in the scene are positioned far from the camera. Something similar also happens with the intensity. 
Therefore, it is important to determine the inferior and superior thresholds which define a minimum and maximum integration time (Fig. 3), $\left[\mathrm{t}_{\min }, \mathrm{t}_{\max }\right]$, to acquire the image of the scene correctly, and compute the distance from the camera suitably.

\section{COMPUTING INTEGRATION TIME}

According to the measures shown in Fig. 2, an analysis which defines when an object is considered far or near is presented. This analysis helps to know whether two thresholds of time integration, $\left[\mathrm{t}_{\min }, \mathrm{t}_{\mathrm{max}}\right]$, are needed or only the minimum threshold, $t_{\min }$, is required in order to guarantee the precise value of the distance and intensity measurements. This analysis is divided into three main stepts.

In the first step, the evolution of the amplitude measurement provided by the camera sensor is calculated. The amplitude is a camera parameter which defines the goodness degree for the range images computed using a specific integration time. This measurement is shown in Fig. 3 for objects located at several distances.

An analysis of Fig. 3 shows how the linearisation level determines the degree of oversaturation. Thus, the curve of amplitude grows quickly until reach an absolute maximum when the camera is near of the object and the curve becomes more linear when the camera move away of the object.

The second step is the computation of the frequency distribution by means of the histogram. The histogram will show the tendency of the amplitude value. Fig. 4 shows how the statistics mode (the value that occurs more frequently) follows a central tendency (average values) when the camera is near the object. On the other hand, when the camera is far from the object, the mode tends to small values.

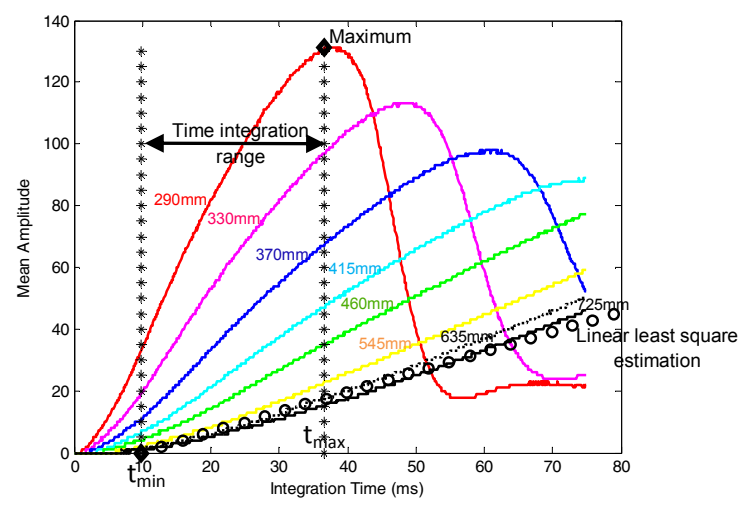

Figure 3. Evolution of mean amplitude, $\mathrm{a}_{\mathrm{m}}$, for the tests of Fig. 2.

An empiric off-line study about the behaviour of several statistical distribution [8] (61 types have been studied), using Kolmogorov-Smirnov (K-S) and Anderson-Darling (A-D) tests, has determined that the frequency histogram (Fig. 4) of the evolution of mean amplitude with respect to integration time (Fig. 3) can be approached by a Johnson SB distribution. In particular, Fig. 4 shows the probability density function (PDF) of Johnson SB adjusted to the sample histogram of several experiments.
Finally, the third step consists of a method to classify the objects between close and far objects. In this step, a threshold of distance which separates both types of objects is computed. As Fig. 5 shows, if the objects are far (distance $>0.4 \mathrm{~m}$ ) the goodness-of-fit using K-S and A-D tests determines correctly the adjustment between the samples and Johnson SB.

However, the adjustment begins to get worse when the camera is moved to positions where the distance to target is small $(<0.4 \mathrm{~m})$. In this case, the K-S and A-D tests determine that the best adjustment is other distribution different from Johnson SB. A look-up-table can be built from the test shown in Fig. 5.

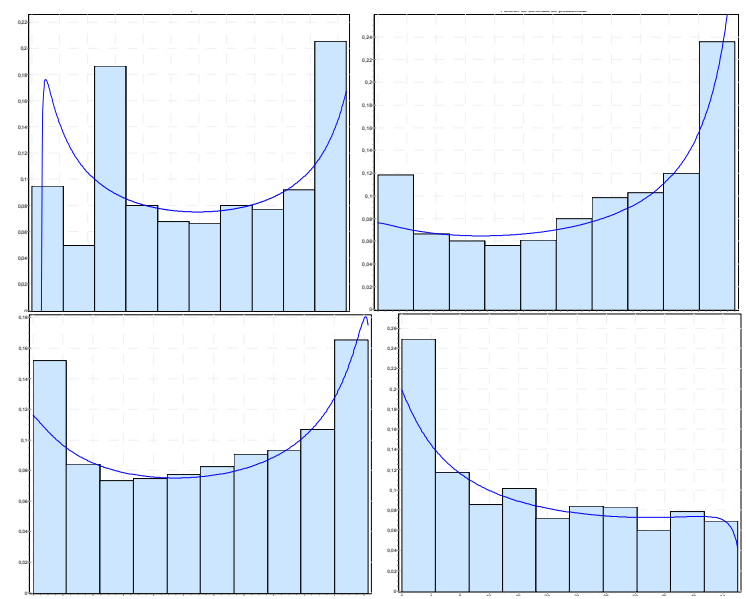

Figure 4. Adjustment of probability densitiy function (PDF)- Johnson SB for amplitude histogram of experiments with distance of 290,370, 460 and 635(mm) shown in Fig. 3.

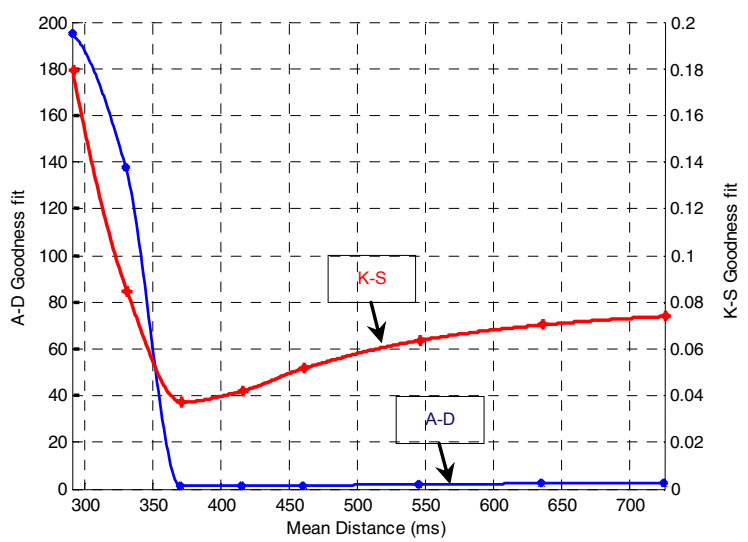

Figure 5. Goodness fit using Kolmogorov-Smirnov (K-S) and AndersonDarling (A-D) tests for the amplitude histograms shown in Fig. 4. 
Fig. 5 illustrates that the Johnson SB distribution is a valid adjustment for distances greater than $0.4 \mathrm{~m}$. Consequently, the distribution analysis of the amplitude samples indicates that this method is a good way to differentiate near objects from far objects when the camera is working in the range distance $<1 \mathrm{~m}$. Thus, if the object is near the maximum amplitude, $\mathrm{t}_{\max }$ is computed and if the object is far, $t_{\min }$ is computed using a linear least square estimation that determines the slope of straight line which fits the amplitude curve (Fig. 3). So the proposed adaptation algorithm for integration time can be summarized in the following lines:

\section{OFFLINE Process}

i. Pose Robot in arbitrary initial location and capture an image, $\mathrm{I}_{\mathrm{t}}$, for some integration time, $\mathrm{t}$ which $\in[0,75] \mathrm{ms}$ and then compute mean Amplitude, $\mathrm{a}_{\mathrm{m}}$ (Fig. 3).

ii. Compute the frequency histogram for $\mathrm{a}_{\mathrm{m}}$ and fit it by means of K-S and A-D Tests in order to classify the scene according to look-up-table of the previous offline analysis (Fig. 5).

- $t_{\min }$ is considered in $10 \mathrm{~ms}(\min \{t\}$ to capture the image at the maximum working distance).

- $t_{\max }$ is computed as the suitable integration time to obtain a desired mean amplitude such as: if (near) $a_{d}=\max \left\{a_{m}\right\}$ else $a_{d}=$ upper quartile $\left(a_{m}\right)$.

\section{ONLINE Process:}

i. Compute the mean amplitude, $a_{m}$, for image, $I_{t}$, captured with an integration time, $\mathrm{t}_{0}$, obtained in the offline process.

ii. Compute deviation error as $e=a_{d}-a_{m}$ where $a_{d}$ is the $\max \left\{\mathrm{a}_{\mathrm{m}}\right\}$ according to a desired minimum distance (see Fig. 3 , step ii of the offline process).

iii. Update integration time following the control law: $t_{k+1}$ $=\mathrm{t}_{\mathrm{k}}+\mathrm{Ket}_{\mathrm{k}}$ where $\mathrm{K}$ is proportional constant and it depends on the robot velocity. Afterwards, return to step i.

The result of the adaptation of integration time from far $(830 \mathrm{~mm})$ to near $(240 \mathrm{~mm})$ is shown in the Fig. 6. In this experiment the robotic arm is moved with constant velocity of $0.01 \mathrm{~m} / \mathrm{s}$. On the one hand, the first part of Fig. 6 shows the computation of the integration time most suitable from the initial location of camera when the robot is kept still. In this case, the proportional constant, $\mathrm{K}$ is equal to 0.005 . On the other hand, the second part of figure shows how the integration time is adapted according the distance when the robot is moving, then $\mathrm{K}$ is 0.00075 .

Moreover, the step 2.iii of the algorithm can be modified so that $\mathrm{t}_{\mathrm{k}}$ can be, always computed depending on the distance between camera and target. Therefore, assuming that some intermediate times, $\mathrm{t}_{\mathrm{k}}$, are known and belonging to $\left[\mathrm{t}_{\min }, \mathrm{t}_{\max }\right]$, the variation of the integration time respect to distance, $\partial \mathrm{t} / \partial \mathrm{d}$, can be computed deriving the polynomial $t(d)$ which is shown in (4).

$$
\begin{aligned}
& t(d)=0,5765 d^{5}+1,1389 d^{4}-1,5270 d^{3}+ \\
& 0,2484 d^{2}+11,8853 d+20,6
\end{aligned}
$$

The intermediate times, $t_{k}$, have been computed in the same way that the integration time for initial location of camera, $\mathrm{t}_{0}$, by means of the algorithm shown previosly. The difference was the locations of the robot which were between the initial and desired location. Afterwards, the computed intermediate times were fitted with the polynomial interpolation given by $\mathrm{t}(\mathrm{d})$.

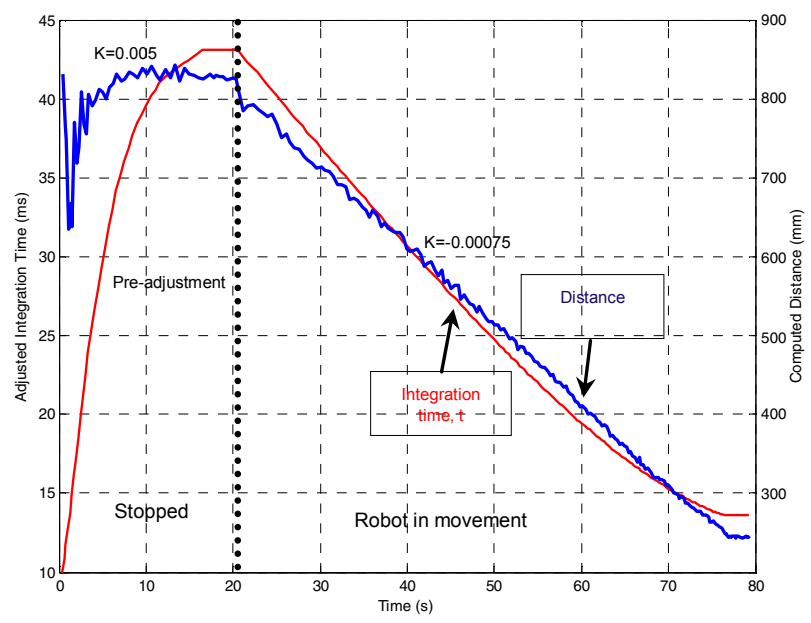

Figure 6. Adaptation of the integration time, t.

\section{CONCLUSIONS}

This work presents an approach to update the integration time of a ToF camera. This method requires two processes. Firstly, an offline process determines the more suitable integration time to begin the robot trajectory from the initial position up to the final (desired) position. Secondly, an online process is used to updtate the integration time according to the velocity of robot movement or according to the variation between integration time and target depth. The method is based on a behaviour analysis of the amplitude parameter of the PMD19k camera but it also can be used with ToF cameras of other manufacturers whenever they compute the amplitude parameter for each pixel.

\section{ACKNOWLEDGMENT}

This work is supported by the Spanish Ministry of Education and Science (MEC) through the research project DPI2008-02647, “Manipulación Inteligente mediante percepción háptica y control visual empleando una estructura articular ubidada en el robot manipulador".

\section{REFERENCES}

[1] M. Frank, M. Plaue, H. Rapp, U. Köthe, B. Jähne and F.A. Hamprecht, "Theoretical and Experimental Error Analysis of Continous-Wave Time-of-Flight Range Cameras," Optical Engineering, vol. 48(1), January 2009, pp. 013602-013618, doi: $10.1117 / 1.3070634$ 
[2] S. Fuchs and G. Hirzinger, "Extrinsic and Depth Calibration of ToFcameras," Proc. IEEE Conference on Computer Vision and Pattern Recognition (CVPR 08), IEEE Press, June 2008, pp. 1-6, doi:10.1109/CVPR.2008.4587828.

[3] P. Khongsab, "Signal Processing and Performance Evaluation of a PMD Camera for Space Docking," Master Thesis Luleâ University of Technology, September 2009.

[4] M. Lindner and A. Kolb, "Calibration of the Intensity-Related Distance Error of the PMD TOF-Camera," Proc. SPIE XXV Conference on Intelligent Robots and Computer Vision, September 2007, pp. 6764-6771, doi: 10.1117/12.752808.

[5] M. Wiedemann, M. Sauer, F. Driewer and K. Schilling, "Analysis and characterization of the PMD camera for application in mobile robotics," Proc. $17^{\text {th }}$ IFAC World Congress (WAC 08), July 2008, pp. 13689-13694.

[6] S. May, B. Werner, H. Surmann and K. Pervölz, "3d time-of-flight cameras for mobile robotics," Proc. IEEE Conference on Intelligent Robots and Systems (IROS 2006), IEEE Press, October 2006, pp. 790-795, doi: 10.1109/IROS.2006.281670.

[7] H. Rapp, "Experimental and Theorical Investigation of Correlating ToF Camera Systems," Master Thesis in Physics. University of Heidelberg, Germany, September 2007.

[8] G. W. Corder and D. I. Foreman, "Nonparametric Statistics for NonStatistician: A Step-by-Step Approach", John Wiley \& Son, New Jersey, 2009. 